\title{
STRONG LOCAL HOMOGENEITY DOES NOT IMPLY COUNTABLE DENSE HOMOGENEITY
}

\author{
JAN VAN MILL
}

\begin{abstract}
We give an example of a connected and locally connected subspace of the plane which is Baire and strongly locally homogeneous (as a consequence, the example is homogeneous) but which is not countable dense homogeneous.
\end{abstract}

1. Introduction. All topological spaces under discussion are separable metric.

A space $X$ is called strongly locally homogeneous if it has an open base $\mathcal{U}$ such that, for each $U \in \mathcal{U}$ and points $x, y \in U$, there exists a homeomorphism $h$ : $X \rightarrow X$ with $h(x)=y$ and $h \mid X \backslash U$ equal to the identity. The most obvious examples of strongly locally homogeneous spaces are locally euclidean spaces and zero-dimensional homogeneous spaces. Clearly, every connected strongly locally homogeneous space is homogeneous.

A space $X$ is called countable dense homogeneous if for any two countable dense subsets $M$ and $N$ of $X$ there is a homeomorphism $f$ of $X$ onto $X$ such that $f(M)=N$. Bennett [2] showed that a connected countable dense homogeneous space is homogeneous.

The relation between countable dense homogeneity and strong local homogeneity is not clear, even in the class of connected spaces. In fact, I do not have an example of a connected countable dense homogeneous space which is not strongly locally homogeneous. Bennett [2] showed that every locally compact space which is strongly locally homogeneous is countable dense homogeneous. This result was generalized by Anderson, Curtis and van Mill [1] who showed that every topologically complete space which is strongly locally homogeneous is countable dense homogeneous. The aim of this paper is to show that the topological completeness assumption in this result is essential. To this end we construct an example of a one-dimensional connected and locally connected subspace of the plane which is strongly locally homogeneous but not countable dense homogeneous. Our example is even Baire which shows that the above cited result of Anderson, Curtis and van Mill is, in a sense, best possible. Since our space is homogeneous it has quite a few homeomorphisms, but since it is not countable dense homogeneous it cannot have too many homeomorphisms. To achieve this we use a method originally due to Kuratowski [6] which was later rediscovered by de Groot [5].

2. Preliminaries. A cardinal is an initial ordinal, and an ordinal is the set of smaller ordinals. $c$ denotes $2^{\aleph_{0}}$.

Received by the editors January 30, 1981.

1980 Mathematics Subject Classification. Primary 54D05, 54F25, 54G20.

(C) 1982 American Mathematical Society $0002-9939 / 82 / 0000-0032 / \$ 02.50$ 
The following classical result, due to Lavrentieff [7], will be important in our construction.

2.1. LemMA. Let $X$ and $Y$ be topologically complete. If $A \subset X$ and $B \subset Y$ and if $h$ : $A \rightarrow B$ is a homeomorphism, then there are $G_{\delta}$-subsets $A^{\prime} \subset X$ and $B^{\prime} \subset Y$ such that $A \subset A^{\prime}$ and $B \subset B^{\prime}$ while moreover $h$ can be extended to a homeomorphism $h^{\prime}$ : $A^{\prime} \rightarrow B^{\prime}$.

The domain and range of a function $f$ will be denoted by $\operatorname{dom}(f)$ and $\operatorname{range}(f)$, respectively. Observe that the collection $\mathscr{F}=\left\{f: \operatorname{dom}(f)\right.$ and $\operatorname{range}(f)$ are $G_{\delta}$-subsets of $\mathbf{R}^{2}$ and $f: \operatorname{dom}(f) \rightarrow \operatorname{range}(f)$ is a homeomorphism $\}$ has cardinality c.

The autohomeomorphism group of $\mathbf{R}^{2}$ will be denoted by $\operatorname{Auth}\left(\mathbf{R}^{2}\right)$. $\mathbf{Q}$ denotes the space of rationals.

3. Construction of the example. Let $\mathcal{H}=\{[a, b] \times[c, d]: a, b, c, d \in \mathbf{Q}, a<b$ and $c<d\}$. For all $H_{0}, H_{1}, H \in \mathcal{H}$ with $H_{0} \cup H_{1} \subset$ int $H$ choose a homeomorphism $h: \mathbf{R}^{2} \rightarrow \mathbf{R}^{2}$ such that

(1) $h\left(H_{0}\right)=H_{1}$, and

(2) $h \mid\left(\mathbf{R}^{2} \backslash H\right)=\mathrm{id}$.

Let $G \subset$ Auth $\left(\mathbf{R}^{2}\right)$ be the set of homeomorphisms obtained in this way. Observe that $G$ is countable. Therefore, the subgroup $\Phi$ of $\operatorname{Auth}\left(\mathbf{R}^{2}\right)$ generated by $G$ is also countable.

For each $x \in \mathbf{R}^{2}$ define

$$
V(x)=\{\varphi(x): \varphi \in \Phi\} .
$$

Observe that $x \in V(x)$, since id $\in \Phi$, and that for all $\varphi \in \Phi$ we have that $\varphi(V(x))=V(x)$. Also, since $\Phi$ is countable, $V(x)$ is countable.

3.1. Lemma. Let $A, B \subset \mathbf{R}^{2}$ be such that $|A|=\mathfrak{c}$ and $|B|<c$. Then $\mid\{x \in A$ : $V(x) \cap B=\varnothing\} \mid=c$.

Proof. Suppose that $|\{x \in A: V(x) \cap B \neq \varnothing\}|=c$. We will derive a contradiction. Since $c$ has uncountable cofinality, i.e. $c$ is not the sum of countably many smaller cardinals, there is a set $A_{0} \subset A$ of cardinality $c$ and a $\varphi \in \Phi$ such that for all $x \in A_{0}$ we have that $\varphi(x) \in B$. Since $\varphi$ is one-to-one and $|B|<c$, this is impossible.

Therefore $|\{x \in A: V(x) \cap B \neq \varnothing\}|<c$ from which follows that $\mid\{x \in A$ : $V(x) \cap B=\varnothing\} \mid=c$.

Let $\mathcal{F}$ be as in $\$ 2$. Put

$$
\mathcal{G}=\{f \in \mathscr{F}:|\{x \in \operatorname{dom}(f): f(x) \notin V(x)\}|=\mathfrak{c}\} .
$$

Then $|\mathcal{G}|=\mathfrak{c}$ (see the proof of Lemma 3.2(a)) and therefore we may list $\mathcal{G}$ as $\left\{f_{\alpha}\right.$ : $\alpha<\mathfrak{c}$. By transfinite induction we will construct for each $\alpha<\mathrm{c}$ a point $x_{\alpha} \in$ $\operatorname{dom}\left(f_{\alpha}\right)$ such that $f_{\alpha}\left(x_{\alpha}\right) \notin V\left(x_{\alpha}\right)$ and

$$
\left(V\left(x_{\alpha}\right) \cup\left\{f_{\alpha}\left(x_{\alpha}\right)\right\}\right) \cap\left(\bigcup_{\beta<\alpha} V\left(x_{\beta}\right) \cup\left\{f_{\beta}\left(x_{\beta}\right)\right\}\right)=\varnothing .
$$


This construction is a triviality. Suppose that the points $x_{\beta}$ for $\beta<\alpha$ have been defined. Put $A=\left\{x \in \operatorname{dom}\left(f_{\alpha}\right): f_{\alpha}(x) \notin V(x)\right\}$. By assumption $|A|=c$. In addition, let

$$
B=\bigcup_{\beta<\alpha} V\left(x_{\beta}\right) \cup\left\{f_{\beta}\left(x_{\beta}\right)\right\}
$$

Then $|B|<c$ since $|\alpha|<c$ and each $V\left(x_{\beta}\right)$ is countable. Therefore, by Lemma 3.1, the set $A_{0}=\{x \in A: V(x) \cap B=\varnothing\}$ has cardinality c. Since $A_{0} \subset \operatorname{dom}\left(f_{\alpha}\right)$ and $f_{\alpha}$ is one-to-one, it is also true that $f_{\alpha}\left(A_{0}\right)$ has cardinality c. Therefore we can find a point $x \in A_{0}$ with $f_{\alpha}(x) \notin B$. Define $x_{\alpha}=x$. It is clear that $x_{\alpha}$ is as required.

We claim that

$$
X=\bigcup_{\alpha<c} V\left(x_{\alpha}\right)
$$

is the example we are looking for.

3.2. LEMMA. (a) If $K \subset \mathbf{R}^{2}$ is uncountable and closed then $K \cap X \neq \varnothing$.

(b) $X$ is connected and locally connected.

(c) $X$ is one dimensional.

(d) If $f \in \mathcal{G}$ then for some $x \in \operatorname{dom}(f) \cap X$ we have that $f(x) \notin X$.

Proof. (a) Without loss of generality, $K$ is a Cantor set. Let $K^{\prime}$ be a Cantor set in $\mathbf{R}^{2}$ not intersecting $\cup_{\varphi \in \Phi} \varphi(K)$ and let $h: K \rightarrow K^{\prime}$ be any homeomorphism. Clearly, $h \in \mathcal{G}$ and therefore, by construction $X \cap \operatorname{dom}(h)=X \cap K \neq \varnothing$. For (b), assume that $X=U \cup V$, where $U$ and $V$ are disjoint, nonempty and open in $X$. Let $U^{\prime}$ and $V^{\prime}$ be open subsets of $\mathbf{R}^{2}$ with $U^{\prime} \cap X=U$ and $V^{\prime} \cap X=V$. Since $X$ is dense in $\mathbf{R}^{2}$, by (a), $U^{\prime} \cap V^{\prime}=\varnothing$ and consequently, $K=\mathbf{R}^{2} \backslash\left(U^{\prime} \cup V^{\prime}\right)$ separates $\mathbf{R}^{2}$. Since $K$ is necessarily uncountable, by (a), $K \cap X \neq \varnothing$. But this is a contradiction since $X \subset U^{\prime} \cup V^{\prime}$. Consequently, $X$ is connected and the same proof shows that $X$ is locally connected. Observe that (d) is a direct consequence of (3). Consequently, (d) implies that $X$ has empty interior. For if $X$ has nonempty interior then there are Cantor sets $K, L \subset X$ with $L \cap \cup_{\varphi \in \Phi} \varphi(K)=\varnothing$. If $h$ : $K \rightarrow L$ is any homeomorphism then $h \in \mathcal{G}$ and since range $(h) \subset X$ this contradicts (d). Therefore, $\operatorname{dim} X \leqslant 1$ [4, 1.8.10]. By connectivity of $X$ we have that $1 \leqslant$ $\operatorname{dim} X$. We conclude that $\operatorname{dim} X=1$ which establishes (c).

We will now show that $X$ is not countable dense homogeneous. First we prove two important lemmas.

3.3. LEMMA. Let $U \subset X$ be open and nonempty and let $\mathscr{D}$ be a family of countably many nowhere dense subsets of $X$. Then $|U \backslash \cup \mathscr{D}|=c$. In particuiar, $X$ is Baire.

Proof. Let - denote the closure operator in $\mathbf{R}^{2}$ and let $U^{\prime} \subset \mathbf{R}^{2}$ be open such that $U^{\prime} \cap X=U$. Put $\mathcal{E}=\{\bar{D}: D \in \mathscr{D}\}$. Since $X$ is dense in $\mathbf{R}^{2}$, by Lemma 3.2(a), each member of $\mathcal{E}$ is nowhere dense in $\mathbf{R}^{2}$. Consequently, $U^{\prime} \backslash \cup \mathcal{E}$ contains a Cantor set, say $K$. This Cantor set, as any Cantor set, contains a family of $c$ pairwise disjoint Cantor sets. Therefore, the desired result follows from Lemma 3.2(a). 
3.4. LEMmA. If $h: X \rightarrow X$ is any homeomorphism, then $|\{x \in X: h(x) \notin V(x)\}|<c$.

Proof. Suppose not. By Lemma 2.1 find $G_{\delta}$ 's $S$ and $T$ containing $X$ such that $h$ can be extended to a homeomorphism $h^{\prime}: S \rightarrow T$. Then $h^{\prime} \in \mathcal{G}$ and therefore, by Lemma 3.2(d), there is an $x \in \operatorname{dom}\left(h^{\prime}\right) \cap X$ with $h^{\prime}(x) \notin X$. Since $\operatorname{dom}\left(h^{\prime}\right) \cap X=$ $X$ and $h^{\prime}$ extends $h$, we find that for some $x \in X$ we have that $h(x) \notin X$, which is a contradiction.

We can now show that $X$ is not countable dense homogeneous.

3.5. ThEOREM. There is a countable dense set $E \subset X$ such that for each homeomorphism $h: X \rightarrow X$ we have that $E \cap h(E) \neq \varnothing$. This implies that $X$ is not countable dense homogeneous.

Proof. Let $D \subset X$ be a countable dense set and put

$$
E=\bigcup_{x \in D} V(x)
$$

Observe that, since $\Phi$ is a subgroup of $\operatorname{Auth}\left(\mathbf{R}^{2}\right), E \subset X$. We claim that $E$ is as required. To this end, let $h: X \rightarrow X$ be a homeomorphism. For each $\varphi \in \Phi$ put $A_{\varphi}=\{x \in X: h(x)=\varphi(x)\}$. Notice that $A_{\varphi}$ is closed in $X$ and that, by Lemma 3.4 ,

$$
\left|X \backslash \bigcup_{\varphi \in \Phi} A_{\varphi}\right|<\mathrm{c} .
$$

Consequently, by Lemma 3.3, there is a $\varphi \in \Phi$ such that $A_{\varphi}$ is not nowhere dense. Since $A_{\varphi}$ is closed, it contains a nonempty open set, and therefore must intersect $E$. Consequently, we can find a point $x \in D$ and an element $\psi \in \Phi$ with $\psi(x) \in A_{\varphi}$. We conclude that

$$
h(\psi(x))=\varphi(\psi(x))=(\varphi \circ \psi)(x) \in V(x) \subset E,
$$

since $\Phi$ is a subgroup of Auth $\left(\mathbf{R}^{2}\right)$. Therefore, $E \cap h(E) \neq \varnothing$.

By Lemma 3.3 there is a countable dense set $F \subset X$ which misses $E$. Clearly, no autohomeomorphism of $X$ can map $E$ onto $F$.

3.6. REMARK. There only remains to prove that $X$ is strongly locally homogeneous. This will be postponed until the next section. If $E \subset X$ is as in the proof of Theorem 3.5 then $E$ has, as we will show, the property that for any countable compact $K \subset X$ there is a homeomorphism $h: X \rightarrow X$ with $h(E \cup K)=E$. Theorem 3.5 shows that the compactness of $K$ is essential. This is very unusual of course.

4. $X$ is strongly locally homogeneous. By Lemma 3.4, $X$ cannot have "many" autohomeomorphisms. However, $X$ has some, since by construction $\varphi(X)=X$ for all $\varphi \in \Phi$. Therefore, if $\varphi \in \Phi$ then $\varphi \mid X$ is an autohomeomorphism of $X$. We will use these countably many homeomorphisms to show that $X$ is strongly locally homogeneous and hence to produce $c$ autohomeomorphisms of $X$. Our technique of proof is inspired by van Mill [8].

First note that, by the special choice of $\Phi$, for any two elements $H_{0}, H_{1} \in \mathcal{K}$ there is an autohomeomorphism $h$ of $X$ with $h\left(H_{0} \cap X\right)=H_{1} \cap X$. This shows 
that for any two points $x, y \in X$ and $\varepsilon>0$ there is an autohomeomorphism $h$ of $X$ with $d(h(x), y)<\varepsilon$.

We claim that if $H \in \mathcal{H}$ and if $x, y \in$ int $H \cap X$ then there is an autohomeomorphism $h: X \rightarrow X$ with $h(x)=y$ and $h \mid(X \backslash H)=\mathrm{id}$. This obviously implies that $X$ is strongly locally homogeneous.

To this end, take $H \in \mathcal{H}$ and $x, y \in$ int $H \cap X$. Without loss of generality we may assume that diam $H<1$. The homeomorphism we are looking for will be of the form $\lim _{n \rightarrow \infty} \psi_{n} \circ \cdots \circ \psi_{1}$, where each $\psi_{n}$ is of the form $\varphi_{n} \mid X$ with $\varphi_{n} \in \Phi$.

For each $n \in \mathbf{N}$ we will construct an element $H_{n} \in \mathcal{H}$ with $y \in$ int $H_{n} \subset H_{n} \subset$ $H$ and a homeomorphism $\psi_{n}: X \rightarrow X$ such that if $V_{n}=$ int $H_{n} \cap X$ then

(1) diam $V_{n}<2^{-(n-1)}$ and $H_{n} \subset$ int $H_{n-1}$;

(2) $x \in \psi_{1}^{-1} \circ \cdots \circ \psi_{n}^{-1}\left(V_{n}\right)$ and diam $\psi_{1}^{-1} \circ \cdots \circ \psi_{n}^{-1}\left(V_{n}\right)<2^{-(n-1)}$;

(3) $\psi_{n} \mid\left(X \backslash H_{n-1}\right)=$ id.

Let $\varphi_{1} \in \Phi$ be such that $d\left(\varphi_{1}(x), y\right)<\frac{1}{2}$ and $\varphi_{1} \mid(X \backslash H)=$ id. Put $\psi_{1}=\varphi_{1} \mid X$ and $H_{1}=H$. Suppose that we have constructed $\psi_{i}$ and $H_{i}$ for all $i<n$. Choose $F_{0} \in \mathcal{H}$ such that $\psi_{n} \circ \cdots \circ \psi_{1}(x) \in$ int $F_{0} \cap X \subset$ int $H_{n}$ while moreover diam $\psi_{1}^{-1} \circ \cdots \circ \psi_{n}^{-1}\left(\right.$ int $\left.F_{0} \cap X\right)<2^{-n}$. In addition, let $F_{1} \in \mathcal{H}$ be such that $y \in$ int $F_{1} \subset F_{1} \subset$ int $H_{n}$ and $\operatorname{diam} F_{1}<2^{-n}$. There is an element $\varphi_{n+1} \in \Phi$ with $\varphi_{n+1}\left(F_{0}\right)=F_{1}$ and $\varphi_{n+1} \mid\left(X \backslash H_{n}\right)=$ id. Define $\psi_{n+1}=\varphi_{n+1} \mid X$ and $H_{n+1}=F_{1}$.

Observe that if $d(p, x)>2^{-(n-2)}$ then $\psi_{n-1} \circ \cdots \circ \psi_{1}(p) \notin H_{n-1}$ and consequently, by (1) and (3),

$$
\psi_{k} \circ \cdots \circ \psi_{1}(p)=\psi_{n-1} \circ \cdots \circ \psi_{1}(p)
$$

for all $k \geqslant n-1$.

This implies that if we define $h: X \rightarrow X$ by $h=\lim _{n \rightarrow \infty} \psi_{n} \circ \cdots \circ \psi_{1}$, then $h$ is well defined. Observe that $h(x)=y$ and $h \mid(X \backslash H)=\mathrm{id}$. The easy check that $h$ is a homeomorphism is left to the reader.

4.1. REMARK. Using the same ideas as in this paper it is easy to verify that there exists for each $n \in\{0,1,2, \ldots, \infty\}$ a homogeneous, strongly locally homogeneous space of dimension $n$ which is not countable dense homogeneous.

4.2. REMARK. It is trivial to adapt the above technique to show that the countable dense subset of $X$ constructed in the proof of Theorem 3.5 has the property claimed in Remark 3.6.

4.3. REMARK. The fact that Lavrentieff's Lemma can be used to construct rigid (= no autohomeomorphisms beyond the identity) spaces is well known; for details see Kuratowski [6]. In this paper we used this lemma to kill certain, but not all, homeomorphisms since we aimed at getting a homogeneous space. That this is possible was suggested by van Douwen [3].

\section{REFERENCES}

1. R. D. Anderson, D. W. Curtis and J. van Mill, A fake topological Hilbert space, Trans. Amer. Math. Soc. (to appear).

2. R. Bennett, Countable dense homogeneous spaces, Fund. Math. 74 (1972), 189-194.

3. E. K. van Douwen, A compact space with a measure that knows which sets are homeomorphic, Adv. in Math. (to appear). 
4. R. Engelking, Dimension theory, Polish Scientific Publishers, Warsaw; North-Holland, Amsterdam, 1978.

5. J. de Groot, Groups represented by homeomorphism groups, Math. Ann. 138 (1959), 80-102.

6. K. Kuratowski, Sur la puissance de l'ensemble des "nombres de dimension" de M. Fréchet, Fund. Math. 8 (1925), 201-208.

7. M. Lavrentieff, Contribution d̀ la théorie des ensembles homéomorphes, Fund. Math. 6 (1924), 149-160.

8. J. van Mill, Homogeneous subsets of the real line (to appear).

Subfaculteit Wiskunde, Vrise Universiteit, Amsterdam, The Netherlands 